\title{
Vulvar Cancer by AJCC v7 Stage
}

National Cancer Institute

\section{Source}

National Cancer Institute. Vulvar Cancer by A/CC v7 Stage. NCI Thesaurus. Code C91203.

A term that refers to the staging of vulvar carcinoma according to the American Joint Committee on Cancer, 7 th edition. 